\title{
Thinking of Medical-Nursing Combined Aged Care Mode Based on Big Data Technology
}

\author{
Yan Hou \\ Qilu Normal College, Jinan, Shandong 250014, China
}

Keywords: big data technology; medical-nursing combined; aged care mode; discussion.

\begin{abstract}
With the continuous development of China's information technology, "Internet+" technology has also been widely used in various industries, which has a very positive significance in promoting the development and progress of industry modernization. Furthermore, the continuous maturation of "Internet+" technology and big data technology also has a profound effect on the development of many traditional industries. The aged care industry is an important part of the development of our traditional industries. With the continuous increase of the aging population at the present stage, the aged care industry has also achieved great research and development, while big data technology plays a very active role in the development of this industry. In this context, related discussion was applied on the medical-nursing combined aged care mode in this paper based on big data technology.
\end{abstract}

\section{Introduction}

With the continuous increasingly deepening of aging degree in China at present, the number of elderly people is also increasing. Relatively speaking, the elderly is more susceptible to physical and mental illnesses, and thus the aged care has also become a problem of people's livelihood that the state highly values. Although the problem of care services for the old-age population in our country has become more and more prominent at the present stage, various new-type modes for aged care based on the information-based background have emerged endlessly with the rapid development of information technology in our country. As the most representative technology of modern information technology, it is feasible to apply big data technology to the actual development of the aged care industry and continuously bring forth new ideas in the aged care mode relying on this technology, to ultimately solve the actual problems of national economy and people's livelihood. Therefore, how to continuously innovate in the current aged care modes in China in the context of big data technology has become an issue that needs to be solved urgently at this stage.

\section{Overview of the Development Direction of the Aged Care Mode in the Context of Big Data Technology}

With the continuous maturity of China's Internet information technology today, big data technology has also been incorporated into the national development strategy and has received corresponding support in terms of policies and funding. Under the influence of policies, China's civil affairs departments have also accelerated the process of related informationization and applied big data technology into the service for the elderly, social assistance, charitable welfare and some other aspects. In this context, the aged care mode in China has also undergone tremendous changes. Specifically, a variety of new aged care modes based on big data technology have been established and widely used. All kinds of signals have told us that, in the process of the development of the aged care mode in China, the most important direction is to use "big data + industry" as the main form and take the advantage of big data technology to establish a new type of aged care mode combined with the actual pension situation of China's at the current stage [1]. 


\section{The Positive Role of Big Data Technology in Promoting the Development of the Aged Care Mode}

\subsection{Big Data Technology Helps Improve the Informatization of Aged Care Services}

A problem prevails in the development of the elderly care institutions in China at the present stage, that is, they present a low degree of informatization and lack a corresponding platform for cooperation and exchange when they provide elderly care services to users. In this way, it is very easy to cause the phenomenon of "Information Isolated Island" in the elderly care service, and it has a very serious impact on improving the efficiency of the aged care institutions and the level of elderly care services [2]. Additively, the phenomenon of "Information Isolated Island" will also affect the sharing of public data on aged care services to a large extent, which not only brings certain difficulties to the governance of relevant government departments, but also affects the long-term effective development of the service industry like medical-nursing combined aged care to a large extent. However, if the big data technology can be applied in the actual aged care mode management to build a service platform of big data cloud, it will have a very profound impact on improving the informationization level of the aged care institutions and the level of elderly care services in China at the present.

\subsection{Big Data Technology is Conducive to the Government's Supervision and Policy Orientation of the Aged Care Mode}

In the actual development of the aged care mode, the application of big data technology has a very positive significance for the innovation of government's supervision and policy orientation of it. Under the traditional aged care mode in China, the government's management of the mode of aged care institutions is mainly based on supervision, and no corresponding regulations and policy guidance have been formulated. In this way, it is very easy to results in a fact that the agents in the aged care structure of our country and their service quality are of varying quality, which has seriously affected the improvement of aged care services in China. The application of big data technology in the aged care mode not only improves the level of government informatization construction to a large extent, but also promotes changes in government functions to a large extent. Furthermore, big data technology also plays a very active role in the supervision of the aged care mode and policy-oriented innovations, and it has a very positive significance in improving the service quality of elderly care institutions.

\subsection{Big Data Technology Helps Promote the Development of the Aged Care Industry}

In the development process of traditional aged care industry, the mode of aged care service is relatively single and its related projects are also kind of single when they come to actual aged care services. Compared with developed western countries, this single aged care service mode has severely constrained the long-term and effective development of China's aged care service industry. With the application of big data technology in the pension service industry, the aged care industry can formulate corresponding development plans for the aged care industry based on big data technology. The application scope of such development schemes is not wide at this stage and the application performance levels are not the same. However, in terms of the development of the aged care industry, they are not only conducive to forming a good competitive atmosphere, but also enrich the project type in China's aged care services, which has a very positive influence on the development of the aged care industry.

\subsection{Big Data Technology is Conducive to the Promotion of Human Needs of the Elderly}

In the actual development of the aged care mode, the relevant aged care institutions can only stand out in the current fierce market competition by constantly improving their own service modes and the level of aged care services based on the actual needs of users. However, in the actual care services for the elderly, although many aged care institutions have enriched their own service projects, they cannot truly meet the actual individual needs of the elderly, such as personalized services, personalized medicine, and so on. As an advanced data processing technology at the present stage, big data technology can not only fully mine the full value of data, but also can realize the analysis and integration of all data, and ultimately discover the inherent laws of data and perform corresponding data processing [3]. Therefore, if big data technology is applied to the development of the pension 
model, it can analyze all the information of the users. Then, the aged care institutions can provide corresponding personalized and high-quality services according to the analysis results of actual life habits, medical information and other relevant data of the elderly, which plays a very positive role in improving the market competitiveness of the elderly care institutions.

\subsection{Big Data Technology Can Improve the Specialized Service Level of the Aged Care Mode}

For a long time, the service level for the elderly in China has not always been particularly high, and an important factor is that the level of professionalization of aged care in China is less than optimal. The nursing personnel of the elderly in China are seriously inadequate and their professional quality is relatively low, which seriously affects the innovation and development of the aged care mode in China. The application of big data technology into the development of the pension mode has a very positive significance for improving the comprehensive quality and quantity of nursing staff through the establishment of an information platform.

\section{Difficulties and Inadequacies of Big Data Technology in Innovation of the Aged Care Mode}

\subsection{The Current Level of Application of Big Data Technology is Low in China}

At the present stage, the big data technology in China plays a very active role in promoting the innovation of the aged care model and constructing the service mode of medical-nursing combined care for the elderly. However, since China's big data technology is still in its early stages of development, there are still significant differences between it and western developed countries in related technologies. Moreover, many related technologies remain in the concept stage in the actual application and have not realized real R\&D and application. In addition, the lack of professionals related to big data is also a very important issue that China must face at this stage.

\subsection{There is a Certain Degree of Difficulty in the Collection of Relevant Public Data about Aged Care in China}

To realize the innovation of the aged care mode through the application of big data technology and continuously improve the overall level of the medical-nursing combined aged care service mode, it is very important to analyze relevant public data in the elderly care field. However, in terms of the openness of aged care data in China, it is difficult to realize the application of big data technology in the aged care field. Influenced by relevant laws, regulations and policies, the degree of openness of aged care data in China is not particularly high, and as a result, big data technology is difficult to be applied into the innovation of actual aged care.

\subsection{The Innovation of Aged Care Mode Based on Big Data Technology Still has Many Defects}

Although China has vigorously promoted the big data strategy at the present stage, it is still only a slogan in the actual application of big data, especially in promoting the innovation of the aged care mode in China. In this way, many related big data technologies cannot be applied into actual innovation at all in the process of the innovation and development of China's medical-nursing combined aged care mode. Only by continuously improving China's big-data-related technologies and combining with the actual needs of the elderly can we eventually achieve the innovation and development of China's aged care mode.

\section{Discussion on the Countermeasures of Solving the Problems of the Aged Care Based on Big Data Technology}

\subsection{Establishing Big Data Center of Aged Care Information and Opening the Acquisition of Relevant Public Aged Care Data}

Data is an extremely important factor to truly play the positive role of big data in solving the problems in the development of the aged care mode at the current stage and to use this as a basis for realizing the mode innovation of medical-nursing combined aged care. It is necessary to carry out mining and analysis of relevant data through big data technology, and then realize that the relevant agencies and government departments fully understand the actual situation of the elderly in China, and thereby to continuously improve their own aged care service mode combining the actual needs of 
users. Therefore, to give full play to the positive role of big data technology, relevant governments and institutions, first, should build a national-level big data center of aged care information in conjunction with actual related information [4]. This will not only continuously improve the informationization level of governments in the aspect of aged care, but also make it possible for more non-governmental organizations to join in the aged care industry, which will have a very positive significance for improving the overall level of China's aged care industry. Secondly, the government should continue to increase the degree of release of data about aged care. At the same time, as the increasingly degree of openness in data, the government and relevant management departments, in conjunction with the actual development situation at the current stage, should also formulate corresponding standards, laws and regulations for the aged care services and make reasonable regulations for the entire market of the pension industry, and thereby achieve the continuous improvement of the standardization of elderly care services on this basis. This is also a necessary condition for the application of big data technology in the mode innovation of medical-nursing combined aged care.

\subsection{Building an Intelligent Community Platform for Medical-Nursing Combined Care Services to Achieve Data Sharing in the Region}

With the deepening of the ageing population in our country, the health care system for the elderly is gradually established, taking the community as the main unit. The main form of this kind of old-age medical and health system is to take the community-related health institutions as the center to regularly check and treat the physical health and mental health of the elderly, based on which to ensure the physical and mental health of the elderly. Therefore, in the innovation process of medical-nursing combined aged care mode, local governments or relevant aged care institutions can take the advantage of big data technology to build an intelligent platform for medical-nursing combined care based on the community health care system for the elderly, and then build community data center on this basis to achieve data sharing. The so-called community-based medical-nursing combined care platform refers to the mode which takes aged care institutions in the community as the basis and organizes relevant medical personnel to regularly visit the elderly in the community through the cooperation with institutions providing aged care services in the community [5]. This mode not only can better realize the mode innovation of medical-nursing combined aged care, but also can provide differentiated and personalized services according to the actual situation of the elderly, which has very positive meaning to the improvement of services for the elderly and the development of medical and health undertakings.

\subsection{Strengthening the Training of Professionals in Aged Care Services}

The shortage of professional talents is also an important factor that restricts the application of big data technology in the present innovation of medical-nursing combined aged care. The professional talents here referred to mainly include two kinds of talents: one is the talent with professional skills of big data in the field of aged care, and the other one is the personnel who can provide professional aged care services [6]. At the present stage, there is a big shortage of both the talents for elderly services and talents with professional skills of big data in the aged care field. Not only that, there is a big deficiency in the professionalism and stability of existing employees. The existence of many factors has severely restricted the development of the aged care industry in China. Therefore, some targeted strategies must be taken to deal with the corresponding issues. First, in the process of the development of the aged care industry, relevant hospitals and aged care institutions should establish training bases for elderly care services and provide professional training for existing employees. Secondly, a corresponding promotion mechanism should be set up, so that qualified nursing staff of medical-nursing combined institutions can be included in the hospital health care management sequence and enjoy a good development platform. Finally, the state should formulate corresponding policies to train a group of big data professional talents who can serve professional medical-nursing combined institutions to continuously improve the current level of innovation of the aged care mode.

To sum up, as the degree of aging continues deepening in our country, the mode of medical-nursing combined aged care has gradually become an important direction for the 
development of modern social aged care services in China. And thus, applying big data technology into the actual development of the medical-nursing combined aged care mode is of greatly positive significance for the innovation of the current service mode of the aged care institutions in China.

\section{References}

[1]. Liu Xiaochun, Li Zheng. Analysis of the Way of Big Data Boosting the Development of Elderly Care Service Model [J]. Sichuan Labour and Social Security, 2017(S2): 15-18.

[2]. Xie Yinong, Xie Ronghua. Research on the Development of Big Data Boosting Pension Industry [J]. China Business \& Trade, 2017(15):146-147.

[3]. Sui Dangchen, Zhang Ting. The Research for "Internet+ Medical-Nursing Combined" Pension Mode Development [J]. Scientific Research on Aging, 2017, 5(05): 60-68.

[4]. Li Jianxia, Song Penglei. An Analysis of China's Medical Care Combined Pension Service Model [J]. Modern Business, 2017(11):191-192.

[5]. Yao Tingting, $\mathrm{Wu}$ Zonghui. Medical care service mode for the elderly in domestic and countermeasures [J]. Health Medicine Research and Practice, 2017, 14(01): 8-10 20.

[6]. Jing Aizhen, Lu Zhining. Internet Plus and Intelligent Management of Pension in the Era of Big Data [J]. Journal of Shijiazhuang Vocational Technology Institute, 2016, 28(05):27-29. 